\title{
SALT HRS discovery of the binary nucleus of the Etched Hourglass Nebula MyCn 18*
}

\author{
Brent Miszalski ${ }^{1,2 \dagger}$, Rajeev Manick ${ }^{3}$, Joanna Mikołajewska ${ }^{4}$, Hans Van Winckel ${ }^{3}$ and Krystian \\ Iłkiewicz $^{4}$ \\ ${ }^{1}$ South African Astronomical Observatory, PO Box 9, Observatory, 7935, South Africa \\ ${ }^{2}$ Southern African Large Telescope Foundation, PO Box 9, Observatory, 7935, South Africa \\ ${ }^{3}$ Instituut voor Sterrenkunde, KU Leuven, Celestijnenlaan 200D bus 2401, B-3001 Leuven, Belgium \\ ${ }^{4}$ Nicolaus Copernicus Astronomical Center, Polish Academy of Sciences, Bartycka 18, PL-00716 Warsaw, Poland
}

\begin{abstract}
The shaping of various morphological features of planetary nebulae $(\mathrm{PNe})$ is increasingly linked to the role of binary central stars. Identifying a binary within a PN offers a powerful tool with which to directly investigate the formation mechanisms behind these features. The Etched Hourglass Nebula, $\mathrm{MyCn}$ 18, is the archetype for several binary-linked morphological features, yet it has no identified binary nucleus. It has the fastest jets seen in a PN of $630 \mathrm{~km} \mathrm{~s}^{-1}$, a central star position offset from the nebula centre, and a bipolar nebula with a very narrow waist. Here we report on the Southern African Large Telescope (SALT) High Resolution Spectrograph (HRS) detection of radial velocity variability in the nucleus of $\mathrm{MyCn} 18$ with an orbital period of $18.15 \pm 0.04$ days and a semi-amplitude of $11.0 \pm 0.3$ $\mathrm{km} \mathrm{s}^{-1}$. Adopting an orbital inclination of $38 \pm 5 \mathrm{deg}$ and a primary mass of $0.6 \pm 0.1 M_{\odot}$ yields a secondary mass of $0.19 \pm 0.05 M_{\odot}$ corresponding to an M5V companion. The detached nature of the binary rules out a classical nova $(\mathrm{CN})$ as the origin of the jets or the offset central star as hypothesised in the literature. Furthermore, scenarios that produce the offset central star during the AGB and that form narrow waist bipolar nebulae result in orbital separations 80-800 times larger than observed in MyCn 18. The inner hourglass and jets may have formed from part of the common envelope ejecta that remained bound to the binary system in a circumbinary disk, whereas the offset central star position may best be explained by proper motion. Detailed simulations of MyCn 18 are encouraged that are compatible with the binary nucleus to further investigate its complex formation history.
\end{abstract}

Keywords: planetary nebulae: individual: MyCn 18 - planetary nebulae: general - binaries: spectroscopic techniques: radial velocites - stars: AGB and post-AGB

\section{INTRODUCTION}

At least 1 in 5 planetary nebulae host a close binary central star with an orbital period of $\sim 1$ day or less (Bond 2000; Miszalski et al. 2009). These binaries have passed through the poorly understood common envelope phase of binary stellar evolution (Ivanova et al. 2013). The binary fraction of planetary nebulae is expected to be considerably higher if binary interactions are a dominant formation channel (De Marco 2009). Binary central stars with intermediate orbital periods of several days to years may contribute another 23-27\% towards the binary fraction (Nie et al. 2012), but it is unclear

* Based on observations made with the Southern African Large Telescope (SALT) under programmes 2016-2-SCI-034 and 2017-1MLT-010.

†E-mail: brent@saao.ac.za whether the small number of discoveries made so far (Van Winckel et al. 2014; Jones et al. 2017; Miszalski et al. 2018) signify the existence of a substantial population of intermediate period binaries. We are systematically searching for this population (Miszalski et al. 2018) with the High Resolution Spectrograph (HRS, Bramall et al. 2010, 2012; Crause et al. 2014) on the Southern African Large Telescope (SALT, Buckley et al. 2006; O'Donoghue et al. 2006). Further details of the scientific motivation behind the survey can be found in Miszalski et al. (2018).

Included in our survey is the young planetary nebula MyCn 18 (PN G307.5-04.9, Mayall \& Cannon 1940), also known as the Etched Hourglass Nebula. The bipolar appearance of MyCn 18 (Fig. 1. Sahai et al. 1999) is the result of an eponymous hourglass-shaped nebula inclined 38 deg to the line of sight (Dayal et al. 2000; 


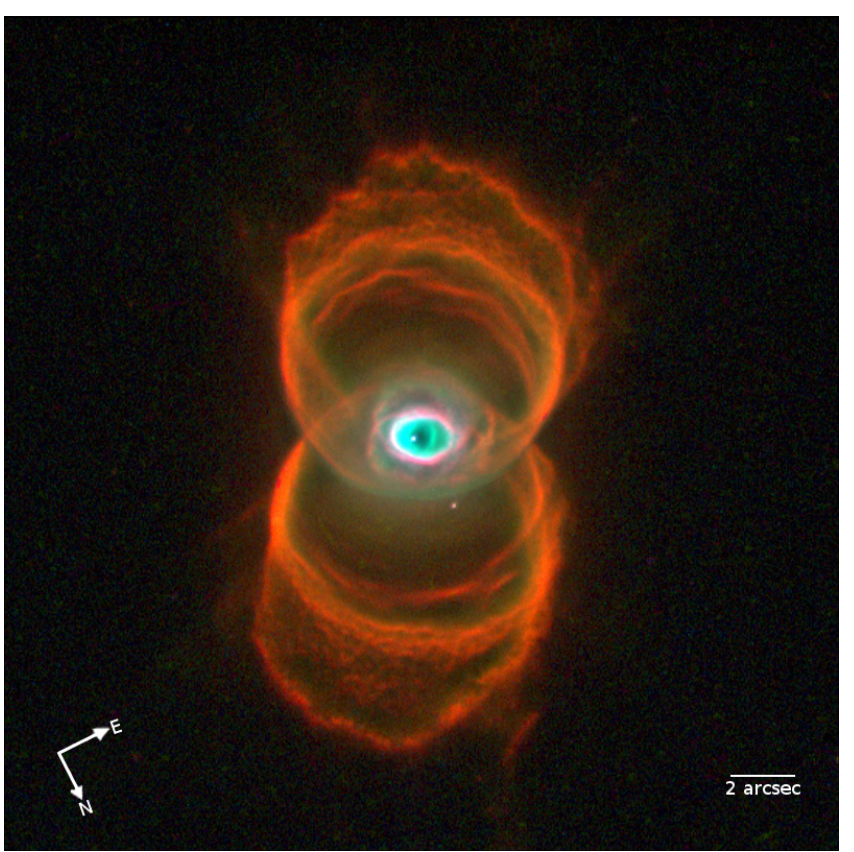

Figure 1. Hubble Space Telescope colour-composite image of MyCn 18 (Sahai et al. 1999) made from F658N (red), F656N (green) and F502N (blue) filters. Image credit: Raghvendra Sahai and John Trauger (JPL), the WFPC2 science team, and NASA.

O'Connor et al. 2000; Clyne et al. 2014). MyCn 18 exhibits several features for which binary interactions are the preferred formation mechanism, yet no evidence has yet been found supporting the presence of a binary companion. These features include polar outflows or jets (Bryce et al. 1997; O'Connor et al. 2000), a central star position offset up to 0.2 " from the geometric centre and its bipolar morphology with a narrow waist (Sahai et al. 1999; Clyne et al. 2014). MyCn 18 has often been compared against bipolar nebulae around low and high mass evolved stars. It is particularly notable for its intriguing nested hourglass structure (Sahai et al. 1999; Clyne et al. 2014) that closely resembles the nebular remnant of SN 1987A (e.g. Burrows et al. 1995; Sugerman et al. 2015), the symbiotic nebula Hen 2-104 (Corradi \& Schwarz 1993; Corradi et al. 2001; Santander-García et al. 2008; Clyne et al. 2015) and $\mathrm{Hb} 12$ which may be a young PN or possibly a symbiotic nebula (Hsia et al. 2006; Kwok \& Hsia 2007; Vaytet et al. 2009; Clark et al. 2014). There are also strong similarities with bipolar and hourglass nebulae around several luminous blue variables (e.g. García-Segura et al. 1999; Smith et al. 2007; Gvaramadze \& Menten 2012; Taylor et al. 2014). Based on these rich connections to several bipolar nebulae, resolving the unknown binary status of $\mathrm{MyCn} 18$ is an important step towards further understanding how bipolar nebulae form.

This paper is structured as follows. Section 2 presents SALT HRS radial velocity monitoring observations of
MyCn 18. We clarify the spectral class of the central star in Sect. 3.1. followed by a description of our radial velocity measurements in Sect. 3.2. We demonstrate the significant periodic variability of these measurements in Sect. 3.3 that prove the binary nature of $\mathrm{MyCn} 18$. Sect. 3.3 also derives the orbital parameters of the binary nucleus. We discuss our results in Sect. 4 in the context of formation scenarios concerning $\mathrm{MyCn} 18$ and other post-CE PNe. We conclude in Sect. 5

\section{SALT HRS OBSERVATIONS}

Multiple observations of the nucleus of $\mathrm{MyCn} 18$ were taken with SALT HRS (Bramall et al. 2010, 2012; Crause et al. 2014), a dual-beam, fibre-fed échelle spectrograph enclosed in a vacuum tank within an insulated, temperature controlled enclosure in the spectrograph room of SALT (Buckley et al. 2006; O'Donoghue et al. 2006). The medium resolution (MR) mode of HRS was used to obtain spectra covering $3700-8900 \AA$ with resolving powers $R=\lambda / \Delta \lambda$ of 43000 and 40000 for the blue and red arms, respectively. During an observation a second fibre, separated at least 20 arcsec from the science fibre, simultaneously observes the sky spectrum. Both object and sky spectra are interleaved on the separate blue $(2 \mathrm{k}$ $\mathrm{x} 4 \mathrm{k}$ ) and red (4k x 4k) CCDs. Regular bias, ThAr arc lamp and quartz lamp flat field calibrations are taken as part of SALT operations.

Table 1 presents a log of the 26 HRS MR observations of $\mathrm{MyCn} 18$ in addition to the radial velocity measurements described in Sect. 3.2 Basic processing of the data was performed by PYSALT (Crawford et al. 2010) before a pipeline based on the MIDAS packages ECHELLE (Ballester 1992) and FEROs (Stahl et al. 1999) developed by A. Y. Kniazev (Kniazev et al. 2016) reduced the data and distributed the data products. The blue spectra which do not feature prominent sky emission lines were not sky subtracted and the red arm spectra were sky subtracted. The small angular size of MyCn 18 and the fibre fed design of HRS precluded the subtraction of nearby nebula emission. The order-merged spectra were converted to a logarithmic wavelength scale using IR A F before heliocentric radial velocity corrections were added using the VELSET task of the RVSAO package (Kurtz \& Mink 1998).

\section{ANALYSIS}

\subsection{Spectral classification}

The spectral classification of the central star of $\mathrm{MyCn} 18$ is unclear in the literature. An $\mathrm{Of}(\mathrm{H})$ classification was made based on an unpublished spectrum (Méndez 1991) and more recently a rare $\mathrm{H}$-deficient $\mathrm{Of}(\mathrm{C})$ classification was made by Lee et al. (2007) that requires a spectrum 'dominated by strong C emissions' (Méndez 1991). We 
Table 1 Observation log of SALT HRS spectra of MyCn 18 and radial velocity measurements. The Julian day represents the midpoint of each exposure and the radial velocity measurements were made from stellar N III $\lambda 4634.14 \AA$ and nebular He I $\lambda 4921.93 \AA$ (see Sect. 3.2).

\begin{tabular}{lcccc}
\hline Julian day & $\begin{array}{c}\text { Exposure time } \\
(\mathrm{s})\end{array}$ & Orbital Phase & $\begin{array}{c}\text { RV }(\mathrm{N} \mathrm{III}) \\
\left(\mathrm{km} \mathrm{s}^{-1}\right)\end{array}$ & $\begin{array}{c}\text { RV }(\mathrm{He}) \\
\left(\mathrm{km} \mathrm{s}^{-1}\right)\end{array}$ \\
\hline 2457847.41564 & 3000 & 0.26 & $-61.67 \pm 0.88$ & $-72.93 \pm 0.06$ \\
2457860.52551 & 3000 & 0.99 & $-73.97 \pm 1.09$ & $-72.45 \pm 0.06$ \\
2457861.48087 & 3000 & 0.04 & $-70.40 \pm 0.97$ & $-72.55 \pm 0.05$ \\
2457880.28752 & 2750 & 0.07 & $-70.29 \pm 2.06$ & $-72.89 \pm 0.07$ \\
2457880.39974 & 2750 & 0.08 & $-68.18 \pm 0.65$ & $-72.75 \pm 0.05$ \\
2457886.28001 & 2750 & 0.40 & $-71.17 \pm 1.09$ & $-72.57 \pm 0.08$ \\
2457892.46150 & 2750 & 0.74 & $-81.86 \pm 0.78$ & $-72.64 \pm 0.06$ \\
2457898.34154 & 2750 & 0.07 & $-65.30 \pm 1.06$ & $-73.19 \pm 0.07$ \\
2457904.22737 & 2750 & 0.39 & $-63.75 \pm 1.21$ & $-73.07 \pm 0.07$ \\
2457910.34825 & 3000 & 0.73 & $-86.15 \pm 1.36$ & $-72.70 \pm 0.06$ \\
2457917.30923 & 2750 & 0.11 & $-65.67 \pm 0.90$ & $-73.18 \pm 0.06$ \\
2457926.28171 & 2500 & 0.60 & $-82.39 \pm 1.91$ & $-72.60 \pm 0.06$ \\
2457934.27854 & 2500 & 0.04 & $-66.21 \pm 1.39$ & $-72.88 \pm 0.06$ \\
2457935.26325 & 3000 & 0.10 & $-64.56 \pm 0.74$ & $-72.69 \pm 0.06$ \\
2457936.25671 & 3000 & 0.15 & $-62.04 \pm 0.91$ & $-72.75 \pm 0.06$ \\
2457938.27615 & 2800 & 0.26 & $-59.02 \pm 0.85$ & $-72.92 \pm 0.07$ \\
2457939.24394 & 2500 & 0.32 & $-60.49 \pm 0.67$ & $-72.77 \pm 0.06$ \\
2457941.30262 & 2500 & 0.43 & $-65.85 \pm 1.36$ & $-72.64 \pm 0.08$ \\
2457942.24390 & 2825 & 0.48 & $-73.50 \pm 0.92$ & $-72.50 \pm 0.07$ \\
2457943.23967 & 2825 & 0.54 & $-68.95 \pm 1.10$ & $-72.52 \pm 0.07$ \\
2457947.30832 & 2825 & 0.76 & $-86.20 \pm 1.28$ & $-72.66 \pm 0.06$ \\
2457949.26982 & 2500 & 0.87 & $-76.58 \pm 0.88$ & $-72.85 \pm 0.06$ \\
2457962.25088 & 2825 & 0.58 & $-78.31 \pm 0.64$ & $-72.94 \pm 0.06$ \\
2457963.25458 & 2825 & 0.64 & $-76.18 \pm 0.79$ & $-72.75 \pm 0.06$ \\
2457965.22577 & 2825 & 0.75 & $-81.75 \pm 0.93$ & $-72.89 \pm 0.06$ \\
2457969.23510 & 2825 & 0.97 & $-74.17 \pm 0.68$ & $-72.89 \pm 0.07$ \\
\hline & & & & \\
\hline
\end{tabular}


created an average blue and red spectrum after shifting all HRS spectra to the rest frame of N III $\lambda 4634 \AA$ using the measurements in Tab. 1 (see Sect. 3.2), IR A F and the RVSAO package (Kurtz \& Mink 1998). Relevant portions are displayed in Fig. 2 which includes a Gaussian fit to He II $\lambda 4686 \AA$ with a full-width at half-maximum (FWHM) of $3.83 \pm 0.03 \AA$ made using the LMFIT package (Newville et al. 2016). The data do not support an $\mathrm{Of}(\mathrm{C})$ classification as the only prominent $\mathrm{C}$ emission features belong to C IV $\lambda 4658,5801,5812 \AA$. The C IV $\lambda 4658 \AA$ emission line is heavily contaminated in our spectrum by nebular [Fe III] emission (Tsamis et al. 2003).

The spectrum exhibits characteristics of both Of and Of-WR stars (Méndez et al. 1990). According to the classification scheme for these stars (Méndez et al. 1990), features supporting an Of classification include the narrow He II $\lambda 4686 \AA$ emission (FWHM $<4.0 \AA$ ) and mildly blueshifted He II $\lambda 4540 \AA$ absorption $(-12.5 \pm 0.7 \mathrm{~km}$ $\mathrm{s}^{-1}$ ), whereas supporting an Of-WR classification there is no absorption at $\mathrm{H} \gamma$ (possibly with a red wing of emission) and blueshifted He II $\lambda 4200 \AA$ more than $-50 \mathrm{~km} \mathrm{~s}^{-1}\left(-52.1 \pm 0.7 \mathrm{~km} \mathrm{~s}^{-1}\right)$. We favour an $\mathrm{Of}(\mathrm{H})$ classification based on the FWHM of He II $\lambda 4686 \AA$ and strong similarities with He II, C IV and N IV features in the $\mathrm{Of}(\mathrm{H})$ central star of M 2-29 (Miszalski et al. 2011a). The temperature of the central star is not well constrained. Gleizes et al. (1989) determined a $\mathrm{H}$ Zanstra temperature of $41 \mathrm{kK}$ and a He II Zanstra temperature of $51 \mathrm{kK}$ based on low-resolution spectra. The stellar He II emission may have been mistaken for nebular He II emission in these spectra since the higher quality HRS spectra rule out any He II nebular emission. Following the example of M 2-29 (Miszalski et al. 2011a), we therefore adopt instead $T_{\text {eff }}=50 \pm 10 \mathrm{kK}$ as He II nebular emission and He I absorption lines are all absent from the HRS spectra.

\section{$3.2 \mathrm{RV}$ measurements}

The radial velocity measurements in Table 1 were determined from the stellar emission feature N III $\lambda 4634.14$ and the nebular emission line He I $\lambda 4921.23$. The L M F I T package (Newville et al. 2016) was used to fit models consisting of a Voigt function and a straight line to N III and a Gaussian function to He I. The fits to N III are displayed in Fig. 3. Uncertainties in each radial velocity measurement are $1 \sigma$ uncertainties determined from the standard error in the fit centroid provided by the fitting routine. The average nebular radial velocity of $-72.78 \pm 0.10 \mathrm{~km} \mathrm{~s}^{-1}$ agrees well with $-71 \mathrm{~km} \mathrm{~s}^{-1}$ measured from spatio-kinematic modeling (Clyne et al. 2014).

\subsection{Periodic variability and orbital parameters}

The SALT HRS radial velocity measurements of the nucleus of MyCn 18 exhibit significant variability compared to the stationary nebular emission (Tab. 1). Period analysis of the radial velocities using a Lomb-Scargle periodogram (Press et al. 1992) reveals the strongest peak to have a period of $18.15 \mathrm{~d}$, significant at the $5 \sigma$ level, while other peaks correspond to the $1-f$ and $1+f$ aliases of this peak (Fig. (4). The $18.15 \mathrm{~d}$ period was used as the basis for a Keplerian orbit model that was built using a least-squares minimization method applied to the phase-folded data. Figure 4 displays the Keplerian orbit fit overlaid on the radial velocity measurements in time and folded with the orbital period. The observed sinusoidal radial velocity shifts prove the binary nature of $\mathrm{MyCn} 18$.

Table 2 presents the orbital parameters of the Keplerian orbit fit determined using Monte Carlo simulations in which the eccentricity was fixed to be zero (for details see Miszalski et al. 2018). The systemic velocity of the or$\operatorname{bit}\left(V_{\text {hel }}=-71.70 \pm 0.24 \mathrm{~km} \mathrm{~s}^{-1} ; V_{\mathrm{LSR}}=-65.20 \pm 0.24\right.$ $\mathrm{km} \mathrm{s}^{-1}$ ) agrees well with the nebular radial velocity of $-71 \mathrm{~km} \mathrm{~s}^{-1}$ (Clyne et al. 2014). This suggests the $\mathrm{N}$ III emission line from the $\mathrm{Of}(\mathrm{H})$-type primary is not significantly disturbed by the stellar wind and traces the motion of the primary.

The estimation of the secondary mass requires some assumptions concerning the orbital inclination $i$ and the primary mass $M_{1}$. Spatio-kinematic studies of post-CE $\mathrm{PNe}$ have demonstrated that the orbital inclination of the binary central star always matches the inclination determined from analysis of the nebula (Hillwig et al. 2016). The inclination of MyCn 18 is well determined to be 38 deg by spatio-kinematic studies (O'Connor et al. 2000; Clyne et al. 2014), although no estimate of the uncertainty was provided by these studies. We have therefore adopted a nominal error of 5 deg in the orbital inclination.

The primary mass $M_{1}$ is more difficult to constrain with the information available in the literature. Masses may be estimated via comparison with post-AGB evolutionary tracks if the temperature and surface gravity or luminosity are known. Determining the surface gravity is complicated by the strong stellar wind which has likely contaminated the absorption line profiles. We therefore adopt an alternative distance-dependent approach that estimates the luminosity of the central star following Sect. 9.4.5 of Frew (2008). We adopt a weighted mean distance of $3092 \pm 507 \mathrm{pc}$, which incorporates distance estimates of $3342 \pm 668$ pc (Stanghellini \& Haywood 2010) and $2750 \pm 780$ pc (Frew et al. 2016), along with an apparent magnitude $m_{V}=14.9 \mathrm{mag}$ (Sahai et al. 1999), $T_{\text {eff }}=50 \pm 10 \mathrm{kK}$ and a reddening of $A_{V}=2.63 \mathrm{mag}$ (Tsamis et al. 2003). We determine using 

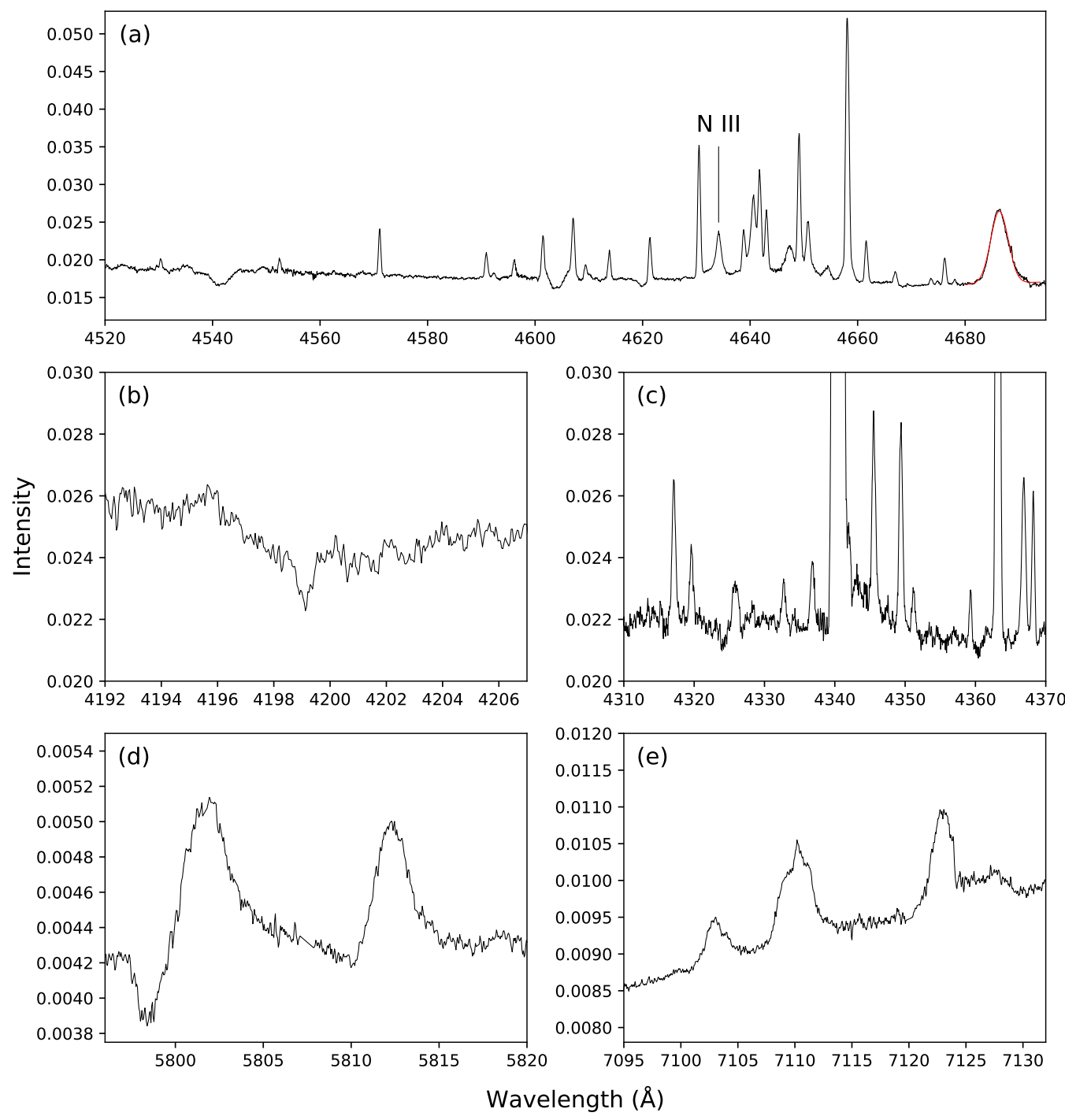

Figure 2. Relevant portions of the average SALT HRS spectrum of the nucleus of MyCn 18. (a) He II $\lambda 4540 \AA$ and $\lambda 4686 \AA$, the latter overlaid with a Gaussian fit (red line, see text). Marked is the N III $\lambda 4634 \AA$ line used to determine radial velocities. (b) He II $\lambda 4200 \AA$, (c) $\mathrm{H} \gamma 4340 \AA$, (d) C IV 5801, $5812 \AA$, (e) N IV $\lambda 7103,7109,7111,7123,7127$ and $7129 \AA$.

Table 2 Orbital parameters of the binary nucleus of MyCn 18.

\begin{tabular}{ll}
\hline Orbital period $P(\mathrm{~d})$ & $18.15 \pm 0.04$ \\
Eccentricity $e$ & 0 (fixed) \\
Radial velocity semi-amplitude $K\left(\mathrm{~km} \mathrm{~s}^{-1}\right)$ & $11.0 \pm 0.3$ \\
Systemic heliocentric velocity $\gamma\left(\mathrm{km} \mathrm{s}^{-1}\right)$ & $-71.70 \pm 0.24$ \\
Separation of primary from centre of mass $a_{1} \sin i(\mathrm{AU})$ & $0.0183 \pm 0.0005$ \\
Mass function $f(m)\left(M_{\odot}\right)$ & $0.0025 \pm 0.0002$ \\
Epoch at radial velocity minimum $T_{0}(\mathrm{~d})$ & $2457965.257 \pm 0.043$ \\
Root-mean-square residuals of Keplerian fit $\left(\mathrm{km} \mathrm{s}^{-1}\right)$ & 2.61 \\
Inclination $i($ degrees) & $38 \pm 5$ \\
Mass of primary $M_{1}\left(M_{\odot}\right)$ & $0.60 \pm 0.10$ \\
Mass of secondary $M_{2}\left(M_{\odot}\right)$ & $0.19 \pm 0.05$ \\
\hline
\end{tabular}


Miszalski et al.

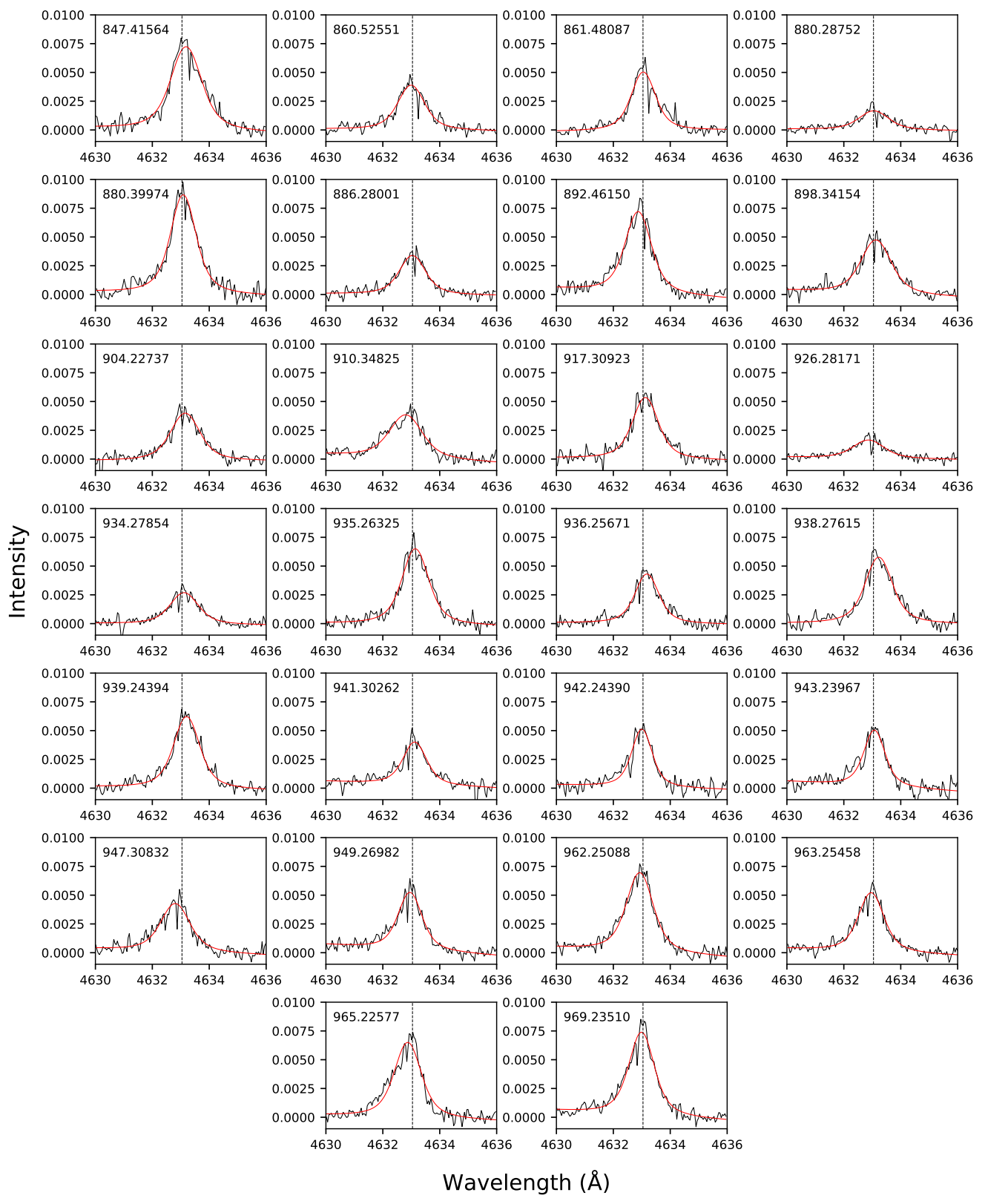

Figure 3. The observed N III $\lambda 4634.14 \AA$ profiles (black lines) were fit with a model composed of a Voigt function and a straight line (red lines). The dashed line represents the expected position of N III at the systemic heliocentric radial velocity of the nebula of $-71 \mathrm{~km}$ $\mathrm{s}^{-1}$ (Clyne et al. 2014). Each panel is labelled with the Julian day of each spectrum minus 2457000 days. 

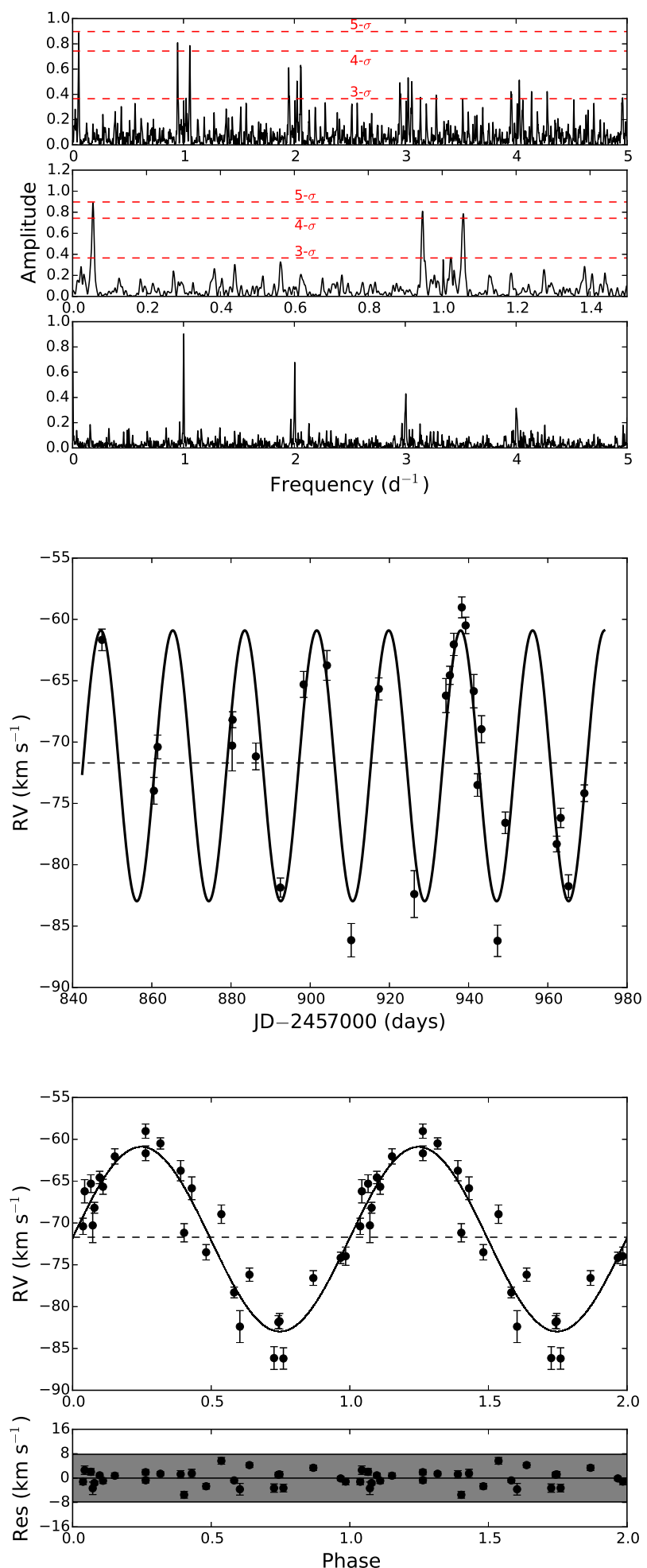

Figure 4. The binary nature of MyCn 18 revealed by SALT HRS. (Top panel) The Lomb-Scargle periodogram of radial velocity measurements (top two segments). The strongest peak at a $5 \sigma$ significance level corresponds to the $18.15 \mathrm{~d}$ orbital period. The $1-f$ and $1+f$ aliases are also visible and the lower segment shows the window function. (Middle and Bottom panels) Radial velocity measurements displayed in time (middle) and folded with the orbital period (bottom). The solid lines represent the Keplerian orbit fit and the shaded region indicates the residuals are within $3 \sigma$ of the fit where $\sigma=2.61 \mathrm{~km} \mathrm{~s}^{-1}$. 
equation 9.13 of Frew (2008) a bolometric correction of $-4.48 \pm 0.66 \mathrm{mag}$ and an absolute bolometric magnitude of $-4.67 \pm 0.77$, where the error in the latter includes errors in the bolometric correction and absolute magnitude added in quadrature which are themselves dominated by the uncertainties in the temperature and the distance, respectively. This results in $\log L / L_{\odot}=3.76 \pm 0.31$ which is typical for young central stars of PNe still on the horizontal part of post-AGB evolutionary tracks. Considering the uncertainties in this distance-dependent method, we adopt $M_{1}=0.6 \pm 0.1 M_{\odot}$ after making comparisons with post-AGB evolutionary tracks (Miller Bertolami et al. 2016). It is unlikely that the mass is above this range as the nebular chemical abundances of $\mathrm{MyCn} 18$ are not consistent with a massive AGB progenitor that has a core mass above $\sim 0.8 M_{\odot}$ (García-Hernández et al. 2016). This is dependent, however, on whether single star AGB models are applicable to post-CE PNe, which is yet to be determined.

The radial velocity semi-amplitude of $K=11.0 \pm$ $0.3 \mathrm{~km} \mathrm{~s}^{-1}, M_{1}=0.6 \pm 0.1 M_{\odot}$ and $i=38 \pm 5 \mathrm{deg}$ therefore gives a secondary mass of $M_{2}=0.19 \pm 0.05 M_{\odot}$. According to the Teff-radius-mass relation for dwarfs with $\mathrm{Z}=0.014$ (Bressan et al. 2012; Chen et al. 2014), the range of masses and temperatures give an M5V companion with an uncertainty of one spectral class (Rajpurohit et al. 2013). The current orbital separation is 0.124 au or $26.6 R_{\odot}$ and the M5V companion has a Roche lobe radius of $\sim 7.5 R_{\odot}$. Even if the radius were inflated by $\sim 2-3$ times as observed in some postCE binaries (Afşar \& İbanoğlu 2008), mass transfer via Roche lobe overflow would not be possible. With the aforementioned distance and reddening the M5V companion would be very faint at $V>21$ mag (Covey et al. 2007), explaining the lack of any secondary features in our spectra. A periodic photometric signal caused by the irradiated atmosphere of the companion may be present with an amplitude of $\sim 0.1 \mathrm{mag}$ (De Marco et al. 2008). No photometric monitoring observations have been reported in the literature, but its detection would be complicated by the bright inner nebula.

\section{DISCUSSION}

\subsection{A classical nova could not have launched the jets of $\mathrm{MyCn} 18$}

The system of collimated outflows or jets of MyCn 18 are remarkable as the fastest observed amongst $\mathrm{PNe}$ with deprojected velocities of up to $630 \mathrm{~km} \mathrm{~s}^{-1}$ (Bryce et al. 1997; O'Connor et al. 2000; Clyne et al. 2014). The jets were ejected $\sim 1050$ years after the main nebula (Clyne et al. 2014). Sahai et al. (1999) estimated the mass of the knots making up the jet system of $\mathrm{MyCn} 18$ to be $\sim 10^{-5}$ $M_{\odot}$. O'Connor et al. (2000) suggested that since classical nova $(\mathrm{CN})$ ejecta have similar masses and kinematics, it may be that a $\mathrm{CN}$ was responsible for producing the jet system of MyCn 18. More recently, Soker \& Kashi (2012) supported the assessment of O'Connor et al. (2000) and further commented that the kinetic energy of the jets was too low to have been formed from an intermediate luminosity optical transient (ILOT) event. Clyne et al. (2014) further speculated on other nova-like formation events, some of which include planetary bodies that may be destroyed. Apart from the knots demonstrating similar mass and velocities to CN ejecta, we emphasise that these hypothetical CN or nova-like scenarios do not have any other observational support. Despite this, these scenarios appear to have been adopted as the de facto explanation for jet formation in MyCn 18 in the literature. As such, it is important to determine whether these hypothetical scenarios are physically compatible with the binary nucleus of $\mathrm{MyCn} 18$. The observed orbital parameters of the binary can be assumed unchanged since the main nebula was ejected at the end of a $\mathrm{CE}$ interaction phase. These parameters uniquely enable us to determine whether any $\mathrm{CN}$ event could have produced the jet system since the main nebula was ejected.

Classical novae occur in cataclysmic variables where the short orbital separation allows for mass transfer and accumulation onto the white dwarf leading to a thermonuclear explosion (Warner 1995). The time needed for the WD to build up a critical layer of hydrogen depends primarily on the mass of the WD and the accretion rate. While models for novae on degenerate WDs in thermal equilibrium are well developed (e.g. Yaron et al. 2005), the same cannot be said for pre-WDs that are found in PNe. Supposing accretion and thermonuclear explosions can occur on pre-WDs (which may also have strong winds), which is currently unclear as no models have explored the topic, then in the following we examine whether a CN could have launched the jets of MyCn 18.

The strictest constraints come from the magnitude of permissible mass transfer rates. In $\mathrm{CNe}$, the primary must have accreted a minimum amount of mass $M_{\text {env }} \sim 10^{-5}-10^{-4} M_{\odot}$ for a $0.6 M_{\odot}$ white dwarf (Yaron et al. 2005) 1 Considering that a $\mathrm{CN}$ launched jet must have accreted mass over the time span between the main nebula and jet formation (only $\sim 1050 \mathrm{yrs}$, Clyne et al. 2014), accreting the above $M_{\text {env }}$ requires that the average accretion rate is an implausible $\dot{M}_{\text {acc }} \sim 10^{-8_{-}}$ $10^{-7} M_{\odot} \mathrm{yr}^{-1}$ that cannot be supplied from the M5V companion. Even if the companion were an active Mdwarf, the accretion rate could only reach a maximum of $\dot{M}_{\text {acc }} \sim 10^{-11} M_{\odot} \mathrm{yr}^{-1}$ during coronal mass ejections (Mullan 1996; Osten \& Wolk 2015), falling far short of the required rate. The inability to achieve sufficiently high accretion onto the primary from the companion

\footnotetext{
${ }^{1}$ Shara et al. (2010) explored very rare luminous red novae that can occur on low-mass WDs $\left(M_{\mathrm{WD}} \lesssim 0.65 M_{\odot}\right)$ accreting at low rates $\left(\dot{M}_{\text {acc }} \sim 10^{-10}-10^{-12} M_{\odot} \mathrm{yr}^{-1}\right.$ ), however they must still accumulate $M_{\mathrm{env}} \sim 10^{-4}-10^{-3} M_{\odot}$ to ignite a nova explosion.
} 
therefore rules out any $\mathrm{CN}$ from powering the jets in $\mathrm{MyCn} 182^{2}$

More generally, it is unlikely that $\mathrm{CNe}$ are in a position to produce jets in post-CE PNe. Jets are extremely rare in cataclysmic variables (Körding et al. 2008, 2011) and are unlikely to account for the large numbers of post-CE $\mathrm{PNe}$ with jets. Furthermore, $\mathrm{CNe}$ are rarely observed to appear in PNe (e.g. Wesson et al. 2008) and the most promising additional candidates have been reclassified as old nova shells (e.g. Miszalski et al. 2016; Shara et al. 2017). In summary, while it is not completely unexpected for a CN to appear within a PN (e.g. Wesson et al. 2008), they are not expected to routinely form jets and are not common enough to play a significant role in producing jets of post-CE PNe.

\subsection{A potential formation scenario for MyCn 18 involving fallback of CE ejecta}

The main hourglass nebula with its narrow waist was ejected about 2700 years ago (Clyne et al. 2014) likely at the end of a CE interaction phase. Preferential deposition of material in the orbital plane during the CE phase likely had a defining influence in shaping the bipolar nebula (e.g. Sandquist et al. 1998; Ricker \& Taam 2012; Passy et al. 2012). The inner hourglass nebula was ejected around 950 years later and was closely followed by the jet system 100 years later (Clyne et al. 2014). The close association in time between the inner hourglass and the jets strongly suggests they were formed from the same reservoir of matter. This matter would likely have been bound to the binary (circumbinary) or either component of the binary. As there could not have been any mass transfer between the binary components (Sect. 3.3), the most likely source of this matter would be bound material left over from the CE phase (Passy et al. 2012; Ricker \& Taam 2012), which simulations suggest can fallback onto the binary or either component to form a disk of a few $\sim 0.1 M_{\odot}$ (Kashi \& Soker 2011; Kuruwita et al. 2016). The observed jet velocities (O'Connor et al. 2000; Clyne et al. 2014) are consistent with being produced by this disk, whether the jets originate near the secondary ${ }^{3}$ the primary (Blackman \& Lucchini 2014), or around both stars. Another more speculative possibility is that a stellar or planetary tertiary companion provides the matter required (e.g. Clyne et al. 2014) $4^{4}$

\footnotetext{
${ }^{2}$ Note that any accretion onto the primary would also be hindered by the strong wind of the primary which can reach $v_{\infty} \sim 1000 \mathrm{~km} \mathrm{~s}^{-1}$ in Of-type central stars (Pauldrach et al. 2004).

${ }^{3}$ An M5 dwarf has an escape velocity of $\sim 582 \mathrm{~km} \mathrm{~s}^{-1}$, assuming $M=0.20 M_{\odot}$ and $R=0.225 R_{\odot}$ (Bressan et al. 2012; Chen et al. 2014).

${ }^{4}$ We emphasise that such scenarios were developed prior to knowledge of the binary system we have discovered in MyCn 18. Any inclusion of a tertiary stellar or planetary companion in formation scenarios concerning $\mathrm{MyCn} 18$ must reproduce the
}

The self-similarity of the inner hourglass with the main nebula (Clyne et al. 2014) suggests the accreted matter may have formed a second $\mathrm{CE}$ (or mini-CE) that would likely lead to the formation of the inner hourglass and jets. Precisely how this process may have occurred in $\mathrm{MyCn} 18$ remains to be determined with the aid of detailed simulations. The process may be similar to that outlined by Soker (2017) in which the formation of a circumbinary disk plays a central role and the jets facilitate the removal of any remaining envelope.

\subsection{The offset binary central star position}

The Hubble Space Telescope imaging of MyCn 18 revealed the central star position to be offset from the geometric centre of each nebula component (Fig. 1. Sahai et al. 1999; Clyne et al. 2014). The largest offset of 0.2 " occurs with respect to the inner hourglass and corresponds to $618 \pm 101$ au at our adopted distance of $3092 \pm 507$ pc. As with the high velocity jets, several scenarios have been hypothesised to explain how this offset was produced in the time between the main nebula was ejected and the jets were launched ( 1050 yr, Clyne et al. 2014). Perhaps the most widely accepted explanation in the literature is that the offset is produced by asymmetric mass loss over long timescales on the AGB (Soker et al. 1998). This scenario appears unlikely to apply as the binary we have discovered has a current orbital separation of 0.124 au or $26.6 R_{\odot}$, which is 80-800 times smaller than the expected final separation range of $\sim 10-100$ au expected according to this scenario. If indeed the offset was produced during the AGB, we would expect a larger observed offset and especially a larger offset with respect to the main nebula.

An explosive CN or nova-like event causing a 'kick' to the binary system to produce the offset has also been suggested by Sahai et al. (1999) and Clyne et al. (2014). As we have discussed (Sect. 4.1), the binary parameters do not allow for a $\mathrm{CN}$ or dwarf nova to form during this timeframe. One possibility may be that the material that formed the inner hourglass (see Sect. 4.2) accreted directly onto the WD and produced a nova-like event $5^{5}$

Perhaps the most promising explanation of the offset could be the proper motion. While Sahai et al. (1999) found this explanation problematic, we note that the direction of the offset matches the direction of the proper motion (Fig. 5) which is $\mu(\alpha)=-11.0 \pm-1.1$ milliarcsec $\mathrm{yr}^{-1}$ and $\mu(\delta)=-2.3 \pm 1.1$ milliarcsec $\mathrm{yr}^{-1}$ (Zacharias et al. 2017). This match alone justifies a more thorough investigation than available data permit and is

\footnotetext{
observed binary system. As our observations do not allow us to determine whether a tertiary component is present, further discussion of the wide variety of such hypothetical scenarios is well beyond the scope of this paper.

${ }^{5}$ It is unclear whether this can occur given the pre-WD nature of the primary which also exhibits strong winds.
} 


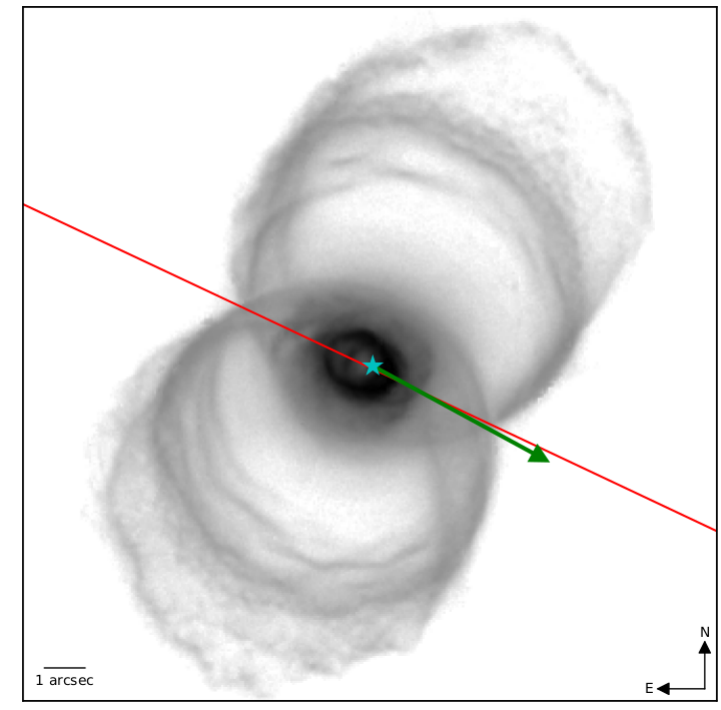

Figure 5. Hubble Space Telescope image of MyCn 18 taken with the $F 656 \mathrm{~N}$ filter showing the direction of proper motion (green arrow) of the central star (cyan star). The red line represents the minor axis.

certainly beyond the scope of this paper. We suggest such a study would involve 3D smoothed particle hydrodynamics $(\mathrm{SPH})$ simulations that include a more comprehensive prescription of the 3D geometry and properties of the inner region of $\mathrm{MyCn} 18^{6}$ as well as higher quality proper motion data anticipated from the Gaia mission.

\subsection{MyCn 18 and other post-CE PNe}

The 18.15 d orbital period of $\mathrm{MyCn} 18$ lies between the $16 \mathrm{~d}$ orbit of NGC 2346 and the $142 \mathrm{~d}$ orbit of NGC 1360 (Miszalski et al. 2018). It is only the sixth binary central star known with a measured orbital period above 10 days (Miszalski et al. 2018). Population synthesis models predict large numbers of multiple day post-CE central stars (e.g. Nie et al. 2012), though it is unclear whether this population exists due to a historical tendency towards photometric surveys to find binary central stars (Miszalski et al. 2018). We will address this question empirically as our ongoing SALT HRS survey progresses, though we can say we have already discovered some new multiple day binaries. The multiple day systems appear to be very rare in the similar population of WD main-sequence binaries that has carefully studied selection effects (Nebot Gómez-Morán et al. 2011). If the $\mathrm{CE}$ phase operates similarly in central stars and WDMS binaries, then the initial SALT HRS discoveries

\footnotetext{
${ }^{6}$ Clyne et al. (2014) considered only two slit positions covering the inner region. Additional slit positions would be beneficial to understand the elusive nature of the inner rings identified by Sahai et al. (1999).
}

might suggest multiple day post-CE binaries are more common than previously thought. Individually, multiple day post-CE central stars may be used to improve our understanding of $\mathrm{CE}$ population synthesis models (e.g. Davis et al. 2010) and simulations of the CE phase involving wider initial orbital separations (e.g. Iaconi et al. 2017).

Whether longer orbital periods favour the formation of bipolar PNe like NGC 2346 and MyCn 18 remains unclear and is currently biased by the small sample size of known binaries. The existence of canonical bipolar post-CE $\mathrm{PNe}$ with much shorter orbital periods, e.g. M 2-19 ( $P=0.67 \mathrm{~d}$, Miszalski et al. 2009) and Hen 2428 ( $P=0.18$ d, Santander-García et al. 2015), suggests there may be no simple correlation between orbital period and morphology. We also note that the often-cited formation scenario for bipolar PNe with narrow waists like MyCn 18 produces final orbital separations of $\sim 10$ $100 \mathrm{au}$ (Soker \& Rappaport 2000), significantly larger than the observed 0.124 au separation of MyCn 18.

$\mathrm{MyCn} 18$ is one of the few post-CE PNe with jets ejected after the $\mathrm{CE}$ phase. Jets ejected after the $\mathrm{CE}$ phase are less common and less understood than those ejected before the CE phase (Tocknell et al. 2014). At present the only other members include NGC 6337 (García-Díaz et al. 2009; Hillwig et al. 2010) and NGC 6778 (Miszalski et al. 2011b; Guerrero \& Miranda 2012). Another poorly understood aspect of post-CE $\mathrm{PNe}$ are those exhibiting high abundance discrepancy factors $(\mathrm{ADF})$ where abundances measured from optical recombination lines are higher than those derived from collisionally excited lines. Tsamis et al. (2004) measured a low $\operatorname{ADF}\left(\mathrm{O}^{2+}\right)$ of 1.8 in MyCn 18. Sowicka et al. (2017) suggested that longer period systems tend to have low measured ADFs based on NGC 5189 (Manick et al. 2015; García-Rojas et al. 2012) and IC 4776 (Sowicka et al. 2017). This depends on the purported 9 d orbital period of the central star of IC 4776 which is not definitive. The orbital period could be much shorter (Sowicka et al. 2017) and more observations are required before IC 4776 can be meaningfully compared against other post-CE PNe.

\section{CONCLUSIONS}

We presented SALT HRS échelle observations of the nucleus of the PN MyCn 18, also known as the Etched Hourglass Nebula. Radial velocity measurements from 26 spectra demonstrate a significant periodic variability of $18.15 \pm 0.04 \mathrm{~d}$. The data prove the presence of a post-CE binary nucleus in MyCn 18 which has long been suspected of being formed by a binary system. Several scenarios concerning the formation of $\mathrm{MyCn} 18$ in the literature are not compatible with the orbital parameters of the binary nucleus. Our main conclusions are as follows: 
- The RV time series measured from the N III $\lambda 4634$ A stellar emission feature demonstrated a periodic variability of $18.15 \pm 0.04 \mathrm{~d}$ at a significance level of $5 \sigma$. The orbital period was used as the basis for a circular Keplerian orbit fit with a semi-amplitude of $11.0 \pm 0.3 \mathrm{~km} \mathrm{~s}^{-1}$ and a systemic velocity of $-71.70 \pm 0.24 \mathrm{~km} \mathrm{~s}^{-1}$. The latter is in good agreement with the nebula systemic velocity $(-71 \mathrm{~km}$ $\mathrm{s}^{-1}$, Clyne et al. 2014). Residuals of the Keplerian fit $\left(2.61 \mathrm{~km} \mathrm{~s}^{-1}\right)$ are relatively high because of the influence of stellar winds in the $\mathrm{Of}(\mathrm{H})$ primary whose classification which we have clarified based on a deep stacked spectrum.

- Assuming a distance of $3092 \pm 507 \mathrm{pc}$, we estimate the luminosity of the primary to be $\log L / L_{\odot}=$ $3.76 \pm 0.31$. At $T_{\text {eff }}=50 \pm 10 \mathrm{kK}$, we estimate the primary mass to be $M_{1}=0.60 \pm 0.10 M_{\odot}$ and adopt an orbital inclination of $i=38 \pm 5$ deg determined from spatio-kinematic studies of the nebula (O'Connor et al. 2000; Clyne et al. 2014). The mass function derived from our observations then yields a secondary mass of $M_{2}=0.19 \pm 0.05 M_{\odot}$ corresponding to an M5 dwarf with an uncertainty of one spectral class.

- We rule out previous hypotheses that the jet system of MyCn 18 (O'Connor et al. 2000) formed as the result of a $\mathrm{CN}$ explosion. The orbital separation of 0.124 au or $26.6 R_{\odot}$ is too large to allow for mass transfer via Roche-lobe overflow. Furthermore, an M5V secondary cannot provide the average accretion rate of $\dot{M}_{\text {acc }} \sim 10^{-8}-10^{-7} M_{\odot} \mathrm{yr}^{-1}$ required to accrete the $M_{\text {env }} \sim 10^{-5}-10^{-4} M_{\odot}$ mass required to form a CN (Yaron et al. 2005) in the $\sim 1050 \mathrm{yr}$ timeframe between the main nebula ejection and when the jets are launched (Clyne et al. 2014).

- An alternative formation scenario for $\mathrm{MyCn} 18$ was proposed whereby material from the $\mathrm{CE}$ ejection that formed the main nebula still bound to the binary settles or falls back to envelop the binary (e.g. Kashi \& Soker 2011; Kuruwita et al. 2016). This may have then lead to the formation of the inner hourglass and jets. The exact process in which this occurs remains uncertain and requires detailed simulations, though it could resemble that described by Soker (2017). There may be other mechanisms that contribute to forming $\mathrm{MyCn} 18$, but these must be compatible with the observed binary system.

- We discussed the 0.2" offset the central star shows with respect to the centre of the inner hourglass. The accepted scenario for producing observable offsets during the AGB (Soker et al. 1998) results in orbital separations 80-800 times larger than the observed separation in MyCn 18. The orbital parameters also rule out a $\mathrm{CN}$ or nova-like event to provide a 'kick' to produce the observed offset (e.g. Sahai et al. 1999; Clyne et al. 2014). We favour the proper motion of MyCn 18 to explain the offset central star, especially since the offset is observed along the minor axis of $\mathrm{MyCn} 18$ in the same direction as the proper motion. Detailed 3D SPH simulations of the motion of $\mathrm{MyCn} 18$ that incorporates anticipated Gaia proper motion data are encouraged to explore this possibility further.

- The discovery of the binary nucleus of MyCn 18 strengthens the long suspected link between bipolar nebulae and interacting binary stars (e.g. Soker \& Rappaport 2000). Characterising binary stars in bipolar nebulae across a wide variety of stellar masses and orbital separations may help shed light on common physical mechanisms behind the massloss histories of bipolar nebulae (e.g. Sugerman et al. 2005). One such common mechanism may be $\mathrm{CE}$ evolution, given the strong resemblance between $\mathrm{MyCn} 18$ (a post-CE PN) and the nebular remnant of SN 1987A (a post-CE merger, Morris \& Podsiadlowski 2009).

\section{ACKNOWLEDGEMENTS}

This paper is based on spectroscopic observations made with the Southern African Large Telescope (SALT) under joint South African-Polish programmes 2016-2-SCI-034 and 20171-MLT-010 (PI: B. Miszalski). Polish participation in SALT is funded by grant No. MNiSW DIR/WK/2016/07. We are grateful to our SALT colleagues for maintaining the telescope facilities and conducting the observations. B. M. acknowledges support from the National Research Foundation (NRF) of South Africa. This study has been supported in part by the Polish MNiSW grant 0136/DIA/2014/43, and NCN grants DEC-2013/10/M/ST9/00086 and 2015/18/A/ST9/00746. H. V. W acknowledges support from the Research Council of the KU Leuven under grant number C14/17/082. We thank the anonymous referee for a helpful and constructive report. This paper also features observations made with the NASA/ESA Hubble Space Telescope, and obtained from the Hubble Legacy Archive, which is a collaboration between the Space Telescope Science Institute (STScI/NASA), the Space Telescope European Coordinating Facility (ST-ECF/ESA) and the Canadian Astronomy Data Centre (CADC/NRC/CSA). IRAF is distributed by the National Optical Astronomy Observatory, which is operated by the Association of Universities for Research in Astronomy (AURA) under a cooperative agreement with the National Science Foundation. B. M. thanks S. Mohamed for discussions and A. Y. Kniazev for making available his HRS pipeline data products.

\section{REFERENCES}

Afşar, M., \& İbanoğlu, C. 2008, MNRAS, 391, 802

Ballester, P. 1992, European Southern Observatory Conference and Workshop Proceedings, 41, 177 
Blackman, E. G., \& Lucchini, S. 2014, MNRAS, 440, L16

Bond, H. E. 2000, Asymmetrical Planetary Nebulae II: From Origins to Microstructures, 199, 115

Bramall, D. G., Sharples, R., Tyas, L., et al. 2010, Proc. SPIE, 7735, 77354F

Bramall, D. G., Schmoll, J., Tyas, L. M. G., et al. 2012, Proc. SPIE, 8446, 84460A

Bressan, A., Marigo, P., Girardi, L., et al. 2012, MNRAS, 427,127

Bryce, M., López, J. A., Holloway, A. J., \& Meaburn, J. 1997, ApJL, 487, L161

Buckley, D. A. H., Swart, G. P., \& Meiring, J. G. 2006, Proc. SPIE, 6267, 62670Z

Burrows, C. J., Krist, J., Hester, J. J., et al. 1995, ApJ, 452,680

Chen, Y., Girardi, L., Bressan, A., et al. 2014, MNRAS, 444,2525

Clark, D. M., López, J. A., Edwards, M. L., \& Winge, C. 2014, AJ, 148, 98

Clyne, N., Redman, M. P., Lloyd, M., et al. 2014, A\&A, 569, A50

Clyne, N., Akras, S., Steffen, W., et al. 2015, A\&A, 582, A60

Corradi, R. L. M., \& Schwarz, H. E. 1993, A\&A, 268, 714

Corradi, R. L. M., Livio, M., Balick, B., Munari, U., \& Schwarz, H. E. 2001, ApJ, 553, 211

Covey, K. R., Ivezić, Ž., Schlegel, D., et al. 2007, AJ, 134,2398

Crause, L. A., Sharples, R. M., Bramall, D. G., et al. 2014, Proc. SPIE, 9147, 91476T

Crawford, S. M., Still, M., Schellart, P., et al. 2010, Proc. SPIE, 7737, 773725

Davis, P. J., Kolb, U., \& Willems, B. 2010, MNRAS, 403, 179

Dayal, A., Sahai, R., Watson, A. M., et al. 2000, AJ, 119,315

De Marco, O., Hillwig, T. C., \& Smith, A. J. 2008, AJ, 136,323

De Marco, O. 2009, PASP, 121, 316

Frew, D. J. 2008, Ph.D. Thesis, Macquarie University

Frew, D. J., Parker, Q. A., \& Bojičić, I. S. 2016, MNRAS, 455,1459

García-Díaz, M. T., Clark, D. M., López, J. A., Steffen, W., \& Richer, M. G. 2009, ApJ, 699, 1633

García-Hernández, D. A., Ventura, P., Delgado-Inglada, G., et al. 2016, MNRAS, 461, 542

García-Rojas, J., Peña, M., Morisset, C., Mesa-Delgado, A., \& Ruiz, M. T. 2012, A\&A, 538, A54

García-Segura, G., Langer, N., Różyczka, M., \& Franco, J. 1999, ApJ, 517, 767

Gleizes, F., Acker, A., \& Stenholm, B. 1989, A\&A, 222, 237
Gvaramadze, V. V., \& Menten, K. M. 2012, A\&A, 541, A7

Hillwig, T. C., Bond, H. E., Afşar, M., \& De Marco, O. 2010, AJ, 140, 319

Hillwig, T. C., Jones, D., De Marco, O., et al. 2016, ApJ, 832,125

Hsia, C. H., Ip, W. H., \& Li, J. Z. 2006, AJ, 131, 3040

Iaconi, R., Reichardt, T., Staff, J., et al. 2017, MNRAS, 464, 4028

Ivanova, N., Justham, S., Chen, X., et al. 2013, A\&ARv, 21,59

Jones, D., Van Winckel, H., Aller, A., Exter, K., \& De Marco, O. 2017, A\&A, 600, L9

Kashi, A., \& Soker, N. 2011, MNRAS, 417, 1466

Körding, E., Rupen, M., Knigge, C., et al. 2008, Science, 320,1318

Körding, E. G., Knigge, C., Tzioumis, T., \& Fender, R. 2011, MNRAS, 418, L129

Kniazev, A. Y., Gvaramadze, V. V., \& Berdnikov, L. N. 2016, MNRAS, 459, 3068

Kurtz, M. J., \& Mink, D. J. 1998, PASP, 110, 934

Kuruwita, R. L., Staff, J., \& De Marco, O. 2016, MNRAS, 461,486

Kwok, S., \& Hsia, C. H. 2007, ApJ, 660, 341

Lee, T.-H., Stanghellini, L., Ferrario, L., \& Wickramasinghe, D. 2007, AJ, 133, 987

Manick, R., Miszalski, B., \& McBride, V. 2015, MNRAS, 448, 1789

Mayall, M. W., \& Cannon, A. J. 1940, Harvard College Observatory Bulletin, 913, 7

Méndez, R. H., Herrero, A., \& Manchado, A. 1990, A\&A, 229,152

Méndez, R. H. 1991, Evolution of Stars: the Photospheric Abundance Connection, 145, 375

Miller Bertolami, M. M. 2016, A\&A, 588, A25

Miszalski, B., Acker, A., Moffat, A. F. J., Parker, Q. A., \& Udalski, A. 2009, A\&A, 496, 813

Miszalski, B., Mikołajewska, J., Köppen, J., et al. 2011a, A\&A, 528, A39

Miszalski, B., Jones, D., Rodríguez-Gil, P., et al. 2011b, A\&A, 531, A158

Miszalski, B., Woudt, P. A., Littlefair, S. P., et al. 2016, MNRAS, 456, 633

Miszalski, B., Manick, R., Mikołajewska, J., et al. 2018, MNRAS, 473, 2275

Morris, T., \& Podsiadlowski, P. 2009, MNRAS, 399, 515

Mullan, D. J. 1996, Cool Stars, Stellar Systems, and the Sun, 109, 461

Nebot Gómez-Morán, A., Gänsicke, B. T., Schreiber, M. R., et al. 2011, A\&A, 536, A43

Newville, M., Stensitzki, T., Allen, D. B., et al. 2016, Astrophysics Source Code Library, ascl:1606.014

Nie, J. D., Wood, P. R., \& Nicholls, C. P. 2012, MNRAS, 423, 2764 
O'Connor, J. A., Redman, M. P., Holloway, A. J., et al. 2000, ApJ, 531, 336

O'Donoghue, D., Buckley, D. A. H., Balona, L. A., et al. 2006, MNRAS, 372, 151

Osten, R. A., \& Wolk, S. J. 2015, ApJ, 809, 79

Passy, J.-C., De Marco, O., Fryer, C. L., et al. 2012, ApJ, 744, 52

Pauldrach, A. W. A., Hoffmann, T. L., \& Méndez, R. H. 2004, A\&A, 419, 1111

Press, W. H., Teukolsky, S. A., Vetterling, W. T., \& Flannery, B. P. 1992, Cambridge: University Press, c1992, 2nd ed.

Rajpurohit, A. S., Reylé, C., Allard, F., et al. 2013, A\&A, 556, A15

Ricker, P. M., \& Taam, R. E. 2012, ApJ, 746, 74

Sahai, R., Dayal, A., Watson, A. M., et al. 1999, AJ, 118,468

Sandquist, E. L., Taam, R. E., Chen, X., Bodenheimer, P., \& Burkert, A. 1998, ApJ, 500, 909

Santander-García, M., Corradi, R. L. M., Mampaso, A., et al. 2008, A\&A, 485, 117

Santander-García, M., Rodríguez-Gil, P., Corradi, R. L. M., et al. 2015, Nature, 519, 63

Shara, M. M., Yaron, O., Prialnik, D., Kovetz, A., \& Zurek, D. 2010, ApJ, 725, 831

Shara, M. M., Iłkiewicz, K., Mikołajewska, J., et al. 2017, Nature, 548, 558

Smith, N., Bally, J., \& Walawender, J. 2007, AJ, 134, 846

Soker, N., Rappaport, S., \& Harpaz, A. 1998, ApJ, 496, 842

Soker, N., \& Rappaport, S. 2000, ApJ, 538, 241

Soker, N., \& Kashi, A. 2012, ApJ, 746, 100

Soker, N. 2017, MNRAS, 471, 4839

Sowicka, P., Jones, D., Corradi, R. L. M., et al. 2017, MNRAS, 471, 3529

Stahl, O., Kaufer, A., \& Tubbesing, S. 1999, Optical and Infrared Spectroscopy of Circumstellar Matter, 188,331

Stanghellini, L., \& Haywood, M. 2010, ApJ, 714, 1096

Sugerman, B. E. K., Crotts, A. P. S., Kunkel, W. E., Heathcote, S. R., \& Lawrence, S. S. 2005, ApJS, 159, 60

Taylor, W. D., Evans, C. J., Simón-Díaz, S., et al. 2014, MNRAS, 442, 1483

Tocknell, J., De Marco, O., \& Wardle, M. 2014, MNRAS, 439, 2014

Tsamis, Y. G., Barlow, M. J., Liu, X.-W., Danziger, I. J., \& Storey, P. J. 2003, MNRAS, 345, 186

Tsamis, Y. G., Barlow, M. J., Liu, X.-W., Storey, P. J., \& Danziger, I. J. 2004, MNRAS, 353, 953

Van Winckel, H., Jorissen, A., Exter, K., et al. 2014, A\&A, 563, L10

Vaytet, N. M. H., Rushton, A. P., Lloyd, M., et al. 2009,
MNRAS, 398, 385

Warner, B. 1995, Cambridge Astrophysics Series, 28

Wesson, R., Barlow, M. J., Corradi, R. L. M., et al. 2008, ApJ, 688, L21

Yaron, O., Prialnik, D., Shara, M. M., \& Kovetz, A. 2005, ApJ, 623, 398

Zacharias, N., Finch, C., \& Frouard, J. 2017, AJ, 153, 166 